partially improved, and one man had made a complete recovery of speech and language. These authors subsequently reported a patient followed from age 3 to 18 years whose language and behavior correlated with abnormalities on the EEG. Improvement coincided with the disappearance of continuous spike wave during sleep and the onset of a unilateral focus (Roulet E, Deonna T et al. Epilepsia 1991;32:495). Paquier PF et al, Rotterdam, The Netherlands, in a follow-up of 6 patients for periods ranging from 3 to 19 years found the outcome of aphasia variable, with slow improvement in 4 , rapid recovery in 1 , and fluctuating course in 1. (Arch Neurol 1992;49:354). (see Progress in Pediatric Neurology I, PNB Publishers, 1991;pp217-218; and Vol II, 1994;pp57-58, for several references and commentary regarding outcome of L-K syndrome).

Landau-Kleffner syndrome (LKS) and electrical status epilepticus during sleep (ESES). Eleven patients with LKS and bitemporal ESES were followed for a mean of 9 yrs and 8 mos at the Neurological Institute, University of Bologna, Italy. Complete recovery of language and cognitive abilities occurred in $18 \%$, and $64 \%$ were mentally retarded. Poor outcome showed some relation to onset of aphasia before 4 years, duration for longer than 1 year, and long-lasting ESES, probably preexisting speech delay. The common origin of LKS and ESES is confirmed by recent functional brain imaging studies. The elimination of ESES appears to be important in prognosis of LKS. (Rossi PG, Parmeggiani A, Posar A et al. Brain Dev March 1999;21:90-98).

\title{
VALPROATE-INDUCED HYPERANDROGENISM IN GIRLS
}

The reproductive endocrine function in 41 girls, 8 to 18 years old, treated with valproate for epilepsy and in 54 healthy control girls was evaluated at the University of Oulu, Finland. Mean serum testosterone concentrations of pubertal and pre- and post-pubertal girls taking valproate were significantly higher than in controls. Hyperandrogenism (serum testosterone levels 2SD above mean control levels) occurred in one third of prepubertal and pubertal valproate treated girls, and more than one-half of postpubertal girls were affected. Postpubertal girls taking valproate were more obese than controls, but the frequency of menstrual irregularities was not increased. (Vainionpaa LK, Rattya J, Knip M et al. Valproate-induced hyperandrogenism during pubertal maturation in girls with epilepsy. Ann Neurol April 1999;45:444-450). (Respond: Dr Vainionpaa, Department of Pediatrics, University of Oulu, FIN-90220 Oulu, Finland).

COMMENT. Monitoring of serum testosterone, height, weight, and Tanner staging of genitalia and pubic hair, during valproate therapy in adolescent girls with epilepsy may be indicated, particularly when antiepileptic medication is required for extended periods, as in juvenile absence and myoclonic epilepsies. In adolescent girls and especially in patients developing hyperandrogenism, a substitute therapy such as lamotrigine should be considered. Hyperandrogenism is a complication of valproate therapy during pubertal maturation of girls with epilepsy, and the frequency of this side effect increases in postpubertal patients.

The University of Oulu investigators have previously reported on the incidence of obesity, polycystic ovaries, and hyperandrogenism in women taking valproate. Fourteen (64\%) of 22 women receiving valproate were affected compared to $9(21 \%)$ of 43 on carbamazepine and $8(19 \%)$ of 43 untreated controls. Polycystic ovarian syndrome, hyperandrogenic chronic anovulation, is characterized by hirsutism and menstrual disorders. (see Progress in Pediatric Neurology II and III, PNB Publ, 1994 \& 1997 for further commentary on endocrine effects of valproate, including pubertal arrest). 
Valproate-induced biochemical abnormalities in pregnancy, including increased excretion of a-ketoglutarate, lactate, pyruvate, and other metabolites, were corrected by treatment with multivitamin supplements given from 13 to 28 weeks' gestation. Fetal head growth, normal up to 30 weeks, was later slowed, and bitemporal narrowing was noted at birth. (Baggot PJ, Kalamarides JA, Shoemaker JD. Epilepsia April 1999;40:512-515). Metabolic abnormalities, possibly related to the teratogenic effects of valproate, can be corrected with high-dose vitamin supplementation.

Valproate-induced male infertility is reported in a previously fertile 32-year-old man with epilepsy treated with valproate monotherapy. A low and abnormal sperm count returned to normal when valproate was discontinued and felbamate was substituted. (Yerby MS, McCoy GB. Epilepsia April 1999;40:520-521).

\section{HYPOGLYCEMIC SEIZURES AND COGNITIVE FUNCTION}

The effect of hypoglycemic seizures on cognitive function was evaluated in 16 children treated for insulin-dependent diabetes mellitus at the Hospital for Sick Children, Toronto, Canada. Mean age at diagnosis was 4.5 years (range 1-11 yrs), and $9(53 \%)$ had suffered hypoglycemic seizures. Psychological evaluation at diagnosis and after 1,3 , and 7 years found significant deteriorations in verbal IQ vocabulary and digit span but not visuospatial skills over 7 years, primarily between 3 and 7 years. Full-scale IQ performance IQ and achievement test scores were unaffected. These selective declines in cognitive function, targeting working memory, were associated with hypoglycemic seizures. Children having seizures had weaker perceptuomotor, attention, memory, and executive processing skills after 7 years treatment for diabetes. (Rovet JF, Ehrlich RM. The effect of hypoglycemic seizures on cognitive function in children with diabetes: A 7-year prospective study. I Pediatr April 1999;134:503-506). (Reprints: Joanne F Rovet PhD, Department of Psychology, The Hospital for Sick Children, 555 University Ave, Toronto, Ontario, Canada M5G1X8).

COMMENT. This long-term, prospective study demonstrates the risks of neurocognitive sequelae attending insulin control of diabetes mellitus and complicated by hypoglycemic seizures in young children. Intensive therapy and over-control of blood glucose should be avoided unless closely monitored to prevent hypoglycemic seizures.

Several articles on the effects of hypoglycemia on the developing brain are included in the April issue of Journal of Pediatrics.

Duvanel CB et al, Lausanne, Switzerland, investigate the long-term effects of neonatal hypoglycemia on brain growth and psychomotor development in small-for-gestational-age preterm infants (L Pediatr 1999;134:492-498). Recurrent episodes of hypoglycemia are correlated with neurodevelopmental and physical growth deficits until 5 years of age. Without close screening of blood glucose, hypoglycemia in the neonate can be overlooked, since classical signs (apnea, seizures, jitteriness, lethargy, etc) may be absent. Rapid correction of even mild hypoglycemia in the neonate is recommended.

Hume R et al, University of Dundee, Scotland, report "Failure to detect preterm infants at risk of hypoglycemia before discharge." ( $\perp$ Pediatr 1999;134:499-502). Fourteen (18\%) of 79 consecutive preterm infants ready for discharge were found to be hypoglycemic $(<47 \mathrm{mg} / \mathrm{dL})$ but without classical symptoms. When a newborn is identified as susceptible to hypoglycemia, feeding regimens should be frequent and regular to avoid hypoglycemic episodes. 